\title{
Patients' views on their experience of the delivery of single-sex accommodation within the endoscopy department: is it worth it?
}

\author{
Elizabeth F Wiseman, ${ }^{1}$ Kelly Chatten, ${ }^{1}$ Sita Shah, ${ }^{1}$ Richard R Keld, ${ }^{1}$ \\ Yeng $S$ Ang ${ }^{1,2}$
}

- Additional material is published online only. To view please visit the journal online (http://dx.doi.org/10.1136/ flgastro-2016-100683).

\section{Department of} Gastroenterology, Royal Albert Edward Infirmary, Wigan, Lancashire, UK

${ }^{2}$ Faculty of Medical and Human Sciences, University of Manchester, Manchester, UK

\section{Correspondence to}

Dr Yeng S Ang, Department of Gastroenterology, Royal Albert Edward Infirmary, Wigan, WN1 2NN, UK; Yeng.Ang@srft.nhs.uk

Received 9 December 2015 Revised 15 February 2016 Accepted 17 February 2016 Published Online First 14 March 2016
CrossMark

To cite: Wiseman EF, Chatten K, Shah S, et al. Frontline Gastroenterology 2017;8:13-18.

\begin{abstract}
Introduction Provision of single-sex accommodation (SSA) in hospitals is a key National Health Service objective. Department of Health policy to eliminate mixed-sex accommodation (MSA) was implemented in our endoscopy department in 2011. We found no published studies of patients' views on MSA in endoscopy units.

Aim We explored patients' views on MSA and their experience of attending our unit at Royal Albert Edward Infirmary (Wigan, UK) since implementation of the SSA policy.
\end{abstract}

Methods Patients attending the endoscopy unit August-October 2012 and February-April 2015 were invited to participate. Views were surveyed using a 10-point questionnaire.

Results 155 patients were included. A minority were aware of national (36\%) or local (39\%) policies regarding MSA provision. Only $20.0 \%$ and $22.9 \%$ reported that they would feel uncomfortable changing behind a curtain or waiting in a gown in a mixed-sex area, respectively. Most of those that felt uncomfortable (81\% and $71 \%$ ) were female, and women ranked importance of SSA significantly higher than men. However, both sexes ranked importance of SSA significantly lower than that of prompt investigation/ treatment. Admissions to an alternative recovery area specifically to maintain SSA compliance reduced from $25 \%$ (2012) to $8 \%$ (2015), following simple measures to improve list efficiency, with corollary reduction in reports of compromised patient experience.

Conclusions SSA is an important healthcare priority for some patients, especially women.
However, most consider prompt investigation/ treatment a much higher priority. Measures to comply with SSA can negatively affect patient experience. However, we demonstrate that simple measures can result in significant improvements in service delivery and patient experience while remaining compliant with SSA guidance.

\section{INTRODUCTION}

The need to improve patient experience and develop a more patient-centred health service has been highlighted by healthcare professionals and politicians in recent years. Empowering patients to contribute in shared decision making ("no decision about me without me') and putting patients' needs first were identified as key objectives for improving the National Health Service (NHS) in 2010. ${ }^{1}$ Completion of the Friends and Family Test, an immediate patient experience feedback tool, was one of the four national Commissioning for Quality and Innovation goals for 2014/2015, demonstrating the commitment of NHS England to continually improve patient experience. $^{2}$

Sharing inpatient treatment areas with patients of the opposite sex can cause significant distress to some patients. ${ }^{3}$ The 2007 Chief Nursing Officer's (CNO's) report into mixed-sex accommodation (MSA) in hospitals highlighted the importance of eliminating MSA as part of the NHS' commitment to maintaining dignity and privacy for all patients and 
concluded that MSA should be eliminated in all but exceptional circumstances. ${ }^{4}$ This was formalised in the 2010/2011 Operating Framework, instructing primary care trusts to ensure that healthcare providers eliminated MSA and to report those failing to comply to the relevant Strategic Health Authorities. ${ }^{5}$ From April 2011 Trusts were required to declare compliance with this policy. Non-compliance incurred financial sanctions ranging from fines of $10 \%-100 \%$ of the service/ procedure cost for patients affected by breaches to withholding funding for organisational level breaches. $^{6}$ Delivery of single-sex accommodation (SSA) continues to be a key issue, recurring in the 2012/2013 Operating Framework as an area where further improvements were expected. ${ }^{7}$

The endoscopy department at Wigan's Royal Albert Edward Infirmary (RAEI) adopted the MSA policy in 2011. However, the unit, which opened in 2004, has only one recovery bay, comprising several bed areas separated by fabric drapes that are closed by patients while changing to ensure privacy. As these arrangements do not meet the criteria for 'separate accommodation' areas to satisfy the SSA policy, changes to working practice were required. Single-sex lists running on separate days were introduced and urgent or specialist procedures (eg, endoscopic ultrasound) for patients of the opposite sex were accommodated by admission/recovery in a separate endoscopy room. To our knowledge there are no published studies on patients' perspectives of the importance of SSA in endoscopy departments. In this study we have explored the views of patients on the importance of SSA in the endoscopy department and their experience of attending our unit since implementation of the MSA policy.

\section{MATERIALS AND METHODS}

All patients attending the endoscopy unit at RAEI during two separate collection periods (cohort A: August-October 2012; cohort B: February-April 2015) were invited to take part in the study by nursing staff during the routine admission process. Patients' views were assessed using a structured, nondisguised, 10-point questionnaire (see online supplementary figure S1), to evaluate various aspects of patient attitudes surrounding MSA for endoscopy services. This was provided on admission and collected before discharge. Questions were framed in either 'Yes/No' answer format, or as a visual analogue rating scale, ranging from 1 (least important) to 10 (most important). All responses were anonymous and participants were made aware that individual responses could not be retrospectively identified. Data were entered into Microsoft Excel for Mac 2011 (V.14.5.2), and statistics performed by Excel and GraphPad Prism for Mac (V.6.0). Unless otherwise stated, comparisons of categorical variables between sexes or cohorts were by Fisher's exact test, and comparisons of patients' visual analogue rankings between groups were by Mann-Whitney $U$ test. All analyses of significance were two-tailed, with $\mathrm{p}<0.05$ considered statistically significant.

\section{RESULTS}

All patients approached completed the questionnaires providing 155 questionnaires in total (68 cohort A; 87 cohort B). Of 124 respondents providing their sex 67 (54\%) were male and 57 (46\%) female, indicating a reasonably equal distribution of sexes. This was similar for both cohorts (women 44\% cohort A; 47\% cohort $\mathrm{B} ; \mathrm{p}=0.853$ ). Views of patients attending for both urgent and routine endoscopic procedures were represented, since 68/155 (44\%) respondents had waited $<2$ weeks for their endoscopy versus $87 / 155$ $(56 \%)$ having waited $>2$ weeks (similar distribution for both cohorts). Overall, only 55/151 (36\%) of patients surveyed were aware of the Department of Health (DOH) recommendations on MSA, with no evidence for a change in level of awareness between the groups surveyed almost 3 years apart $(\mathrm{p}=0.394)$; by contrast, whereas in 2012 only 16/68 (24\%) patients were aware of the local RAEI endoscopy department policy on MSA, in 2015 this had significantly increased to $43 / 85(51 \%, \mathrm{p}=0.0008)$.

Next we explored patients' tolerance of MSA in the admission/recovery areas of the endoscopy department (figure 1). The majority of patients stated that they would not feel uncomfortable changing behind a curtain $(80 \%)$ or waiting in a gown $(77 \%)$ in an MSA (figure 1A, B). Among those who reported they would feel uncomfortable changing behind a curtain, or waiting in a gown in a mixed-sex area $(20 \%$, $23 \%$, respectively), the significant majority were female $(80 \% \quad(\mathrm{p}=0.0001)$ and $71 \% \quad(\mathrm{p}=0.0025)$, respectively).

Next, we explored attitudes regarding the importance of SSA provision for patients attending the endoscopy department (figures 2 and 3). Patients ranked the importance of SSA provision significantly lower than the importance of access to prompt investigation and treatment (median: 4 (IQR 1-8) vs 10 (IQR 10-10), p<0.0001, Wilcoxon matched-pairs signed rank test; figure 2). When stratified by sex, male patients ranked the importance of SSA significantly lower than female patients (median: 1 (IQR $1-5)$ vs 6 (IQR $1-10)$ respectively, $\mathrm{p}<0.0001$ ) (figure $3 \mathrm{~A}, \mathrm{~B})$. However, both genders rated prompt investigation and treatment as the more important parameter, with no significant difference between men and women (median rank 10 (IQR 9.5-10) vs 10 (IQR $10-10), p=0.255$ ) (figure $3 \mathrm{~A}$ ).

Although the overall median rank allocated to importance of SSA increased from 3 (2012) to 5 (2015), there was no evidence of a significant change in attitude between the two cohorts (data not shown; $\mathrm{p}=0.971$ ). Interestingly, there was a suggestion that 
A

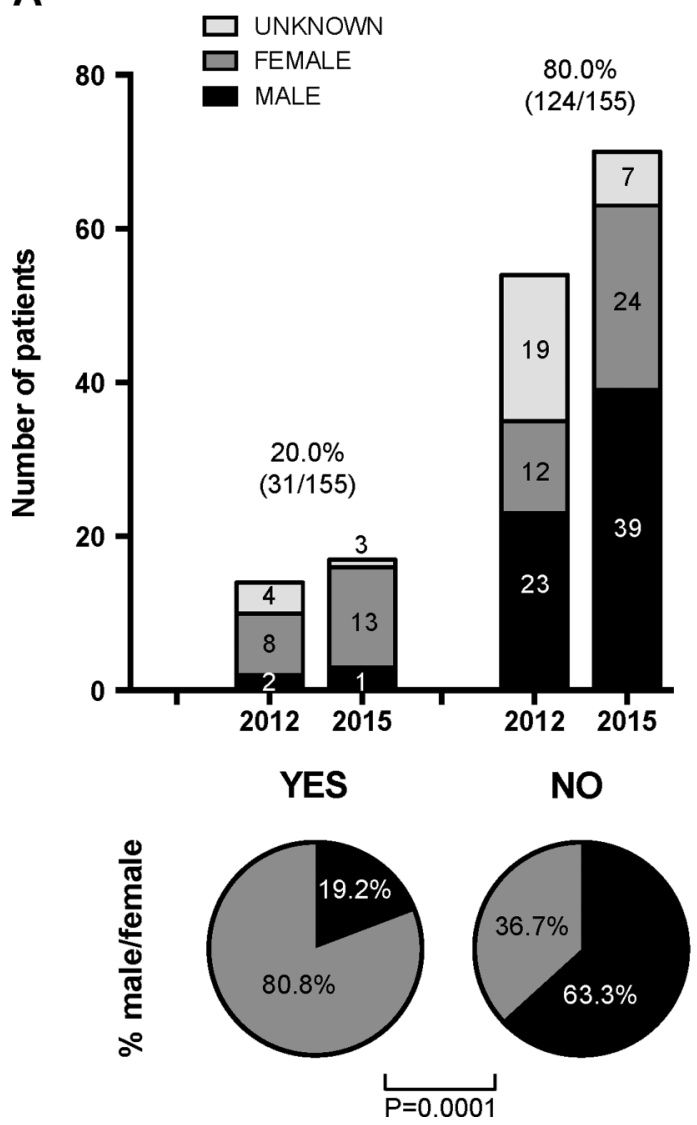

B

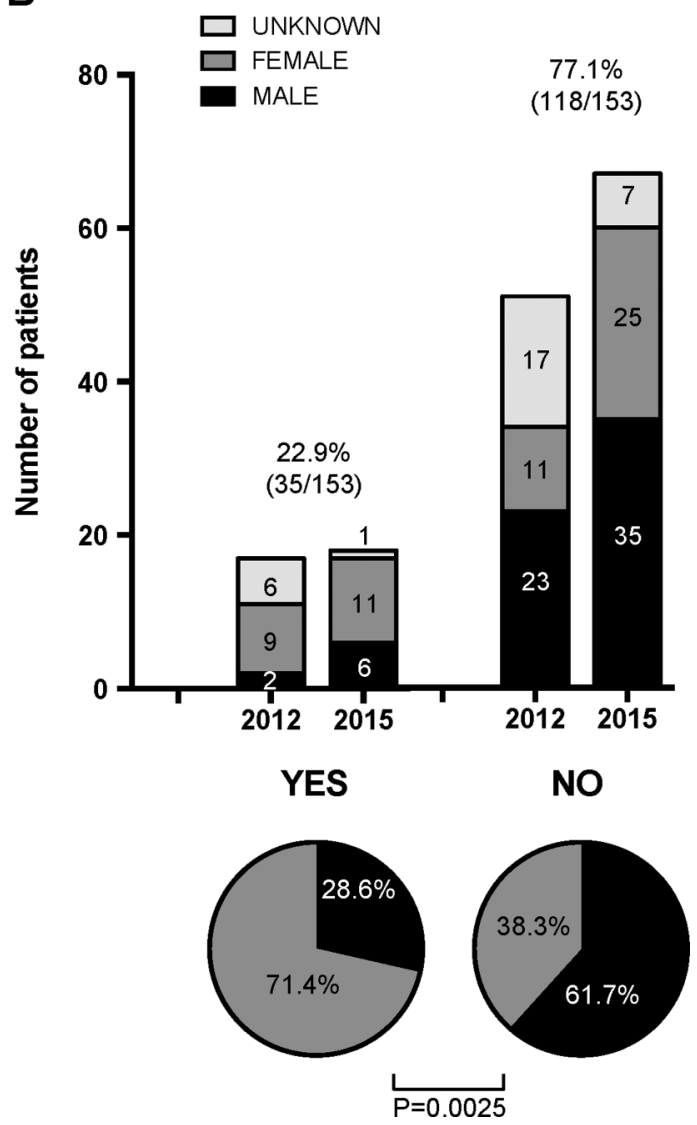

Figure 1 Patients' views when asked would you feel uncomfortable/vulnerable (A) getting changed behind a curtain and (B) waiting in a gown, if there were other patients of the opposite sex in the same area. Bar charts show the number of patients reporting they would ('YES') or would not ('NO') be uncomfortable in each situation; left bars are for patients surveyed in cohort A (2012), right bars for those surveyed in cohort B (2015). Pie charts below each column depict the proportion of male and female patients (where sex provided; cohorts $A$ and $B$ combined). $p$ Values indicate significance of any difference in response by sex (cohorts $A$ and $B$ combined), and are derived from contingency table comparison and Fisher's exact test.

both male and female attitudes might have shifted slightly, but in opposite directions, with men overall allocating slightly higher importance in the more recent cohort (2012 median 1 (IQR 1-2) vs 2015 median 1 (IQR 1-5), p=0.04) and women conversely demonstrating a (non-significant) tendency towards allocating lower importance (2012 median 8.5 (IQR 4.5-10) vs 2015 median 5 (IQR 1-9), $\mathrm{p}=0.14$ ).

In order to comply with the SSA policy the RAEI endoscopy department adopted single-sex lists. Urgent or specialist procedures for patients of the opposite sex to the list running were accommodated by admission/recovery in a separate endoscopy room, rather than the main admission/recovery area. We therefore evaluated the number of patients admitted to an area other than the main admission/recovery area in both cohorts. In cohort A (2012), a sizeable proportion of patients $(17 / 68(25 \%))$ was admitted to an alternative area, and of these patients 7 (41\%) felt that their care was compromised or patient experience diminished as a result. In response to these findings substantial improvements to list planning efficiency were achieved following the appointment of a designated endoscopy unit manager. When audited again in 2015 (cohort B), only 7/87 (8\%) of patients were admitted to an alternative area $(p=0.006)$, and only two patients reported a negative impact on their care or patient experience as a result, indicating evidence of a significant improvement in the requirement for admissions to alternative areas in order to maintain compliance with SSA policy.

\section{DISCUSSION}

Provision of SSA has been highlighted as a priority for healthcare providers within the NHS and was formally mandated in the 2010/2011 Operating Framework. ${ }^{5}$ However, its importance to patients remains unclear. For the 2007 CNO's report on MSA, $>2000$ patients were asked about their experiences and expectations of inpatient care, including their views on MSA. ${ }^{4}$ MSA was less acceptable for women, older persons and patients attending for elective procedures, sex-specific procedures or those who expected a long inpatient stay. However, other aspects of care including good staff attitudes, cleanliness and decent food were ranked as much more important. ${ }^{4} \mathrm{~A}$ 
A

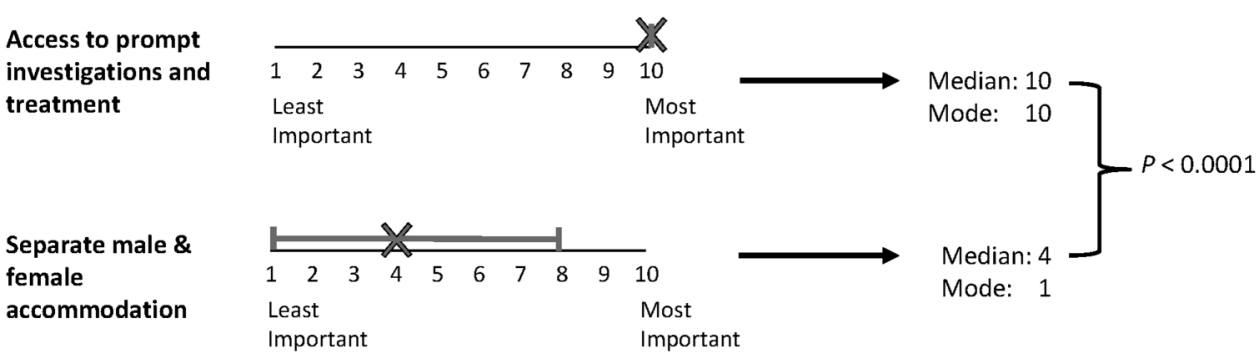

B

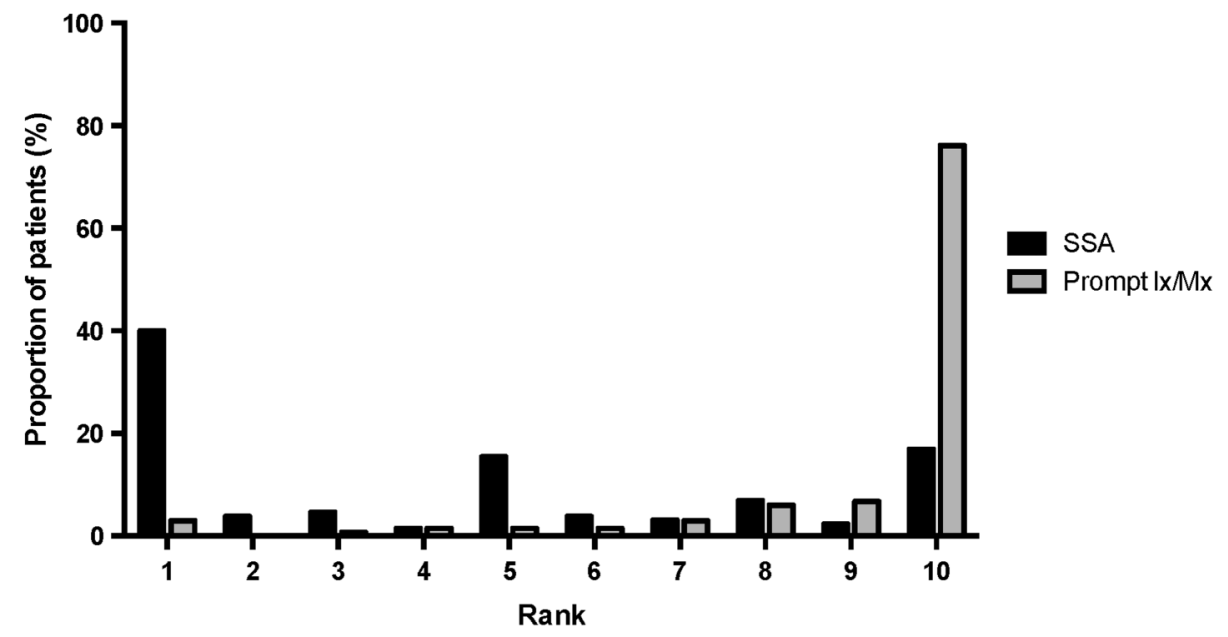

Figure 2 (A) Visual depiction of all patients' ranking of the importance of access to prompt investigations/treatment and the provision of separate male and female accommodation in endoscopy. Median and modal rankings are shown for each feature. Statistical significance of the difference between median ranks for each feature is shown and calculated using the Wilcoxon matched-pairs signed rank test. Crosses on the visual analogue scale represent the median rank for each feature; whiskers indicate the IQR. (B) Bar chart showing the proportion of total patients selecting each rank (1=least important, 10=most important) for each feature. Ix, investigations; Mx, management; SSA, single-sex accommodation.

Picker Institute survey asking patients to rank the importance of 82 aspects of inpatient experience had similar findings: the statement 'I do not need to share a sleeping area with patients of the opposite sex' was ranked 62nd overall (49th for women and 72nd for men). ${ }^{4}$

Patients admitted to hospital in need of urgent treatment are generally more tolerant of MSA. ${ }^{4}$ However, studies of patients' views on acceptability of MSA in medical admission units have been contradictory. One found that 39\% of patients exposed to MSA felt their privacy and dignity were compromised. ${ }^{8}$ Conversely in another, $87 \%$ were comfortable sharing with the opposite sex and $97 \%$ felt that they had sufficient privacy despite lack of definitive SSA. Furthermore, when offered the option of MSA to facilitate a shorter stay, $86 \%$ responded positively. ${ }^{9}$ Similarly, Bryant and Adams reported that both male and female patients would tolerate admission to a mixed-sex surgical bay if it meant immediate treatment. ${ }^{10}$

Opinions on MSA acceptability differ depending on the reason for admission, with patients attending for elective or sex-specific procedures more inclined to prefer SSA. ${ }^{4}$ For example, among patients undergoing urological procedures requirement for equipment such as catheters already compromises privacy and dignity, and in the urology ward 5/12 patients expressed strong negative views on MSA. Although most were older and female, some younger and male patients also reported unease with this. ${ }^{11}$ However, circumstances exist in which allowing MSA may be beneficial. For example, on a renal transplant unit $81 \%$ preferred to be nursed together with donor/ recipient family members. ${ }^{12}$ Thus, despite some common themes (eg, older people and women less tolerant of MSA) patients' views on the importance of SSA are varied and are often context-specific and patient group-specific.

For endoscopy units, where most procedures are performed on an elective basis, the Operating Framework stipulated that breaches should rarely be acceptable. ${ }^{6}$ A focus on maintaining privacy and dignity is particularly important, since some patients are required to change into hospital gowns before waiting with other patients, potentially exacerbating a sense of vulnerability while anticipating an invasive procedure.

To our knowledge this is the first study of patients' views on MSA in the endoscopy department. 
A investigations and treatment
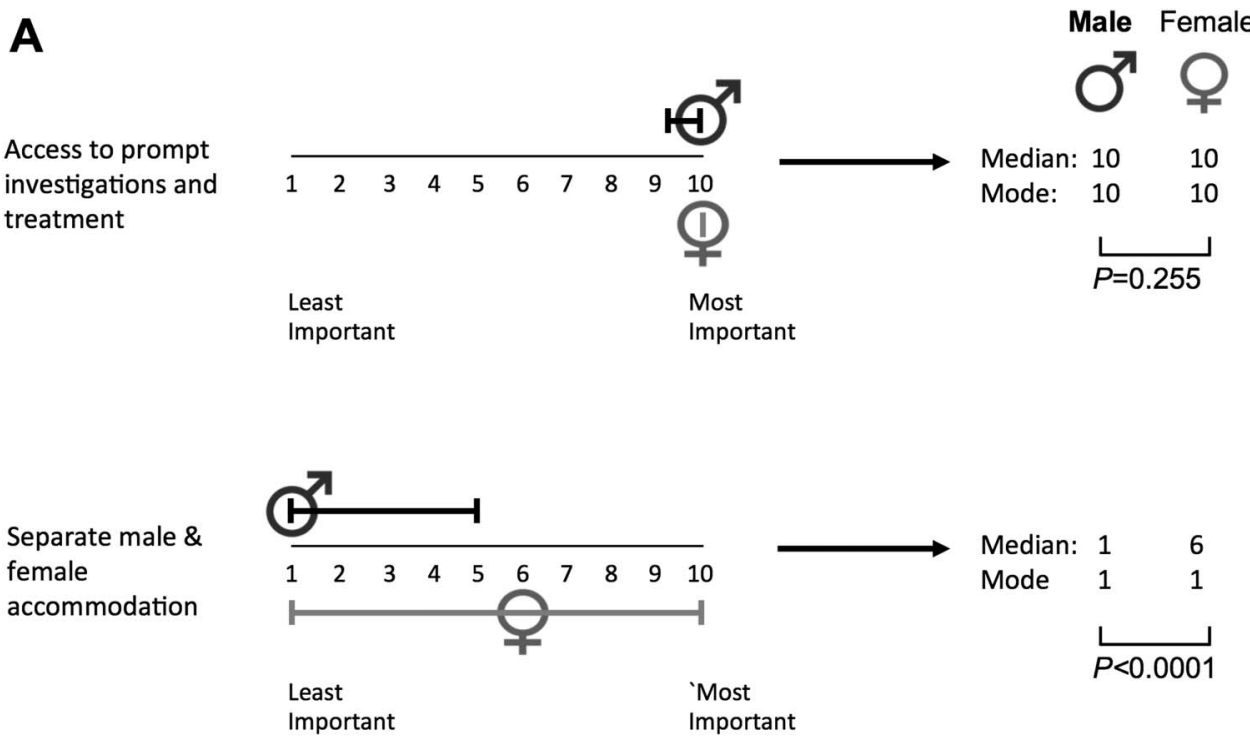

B

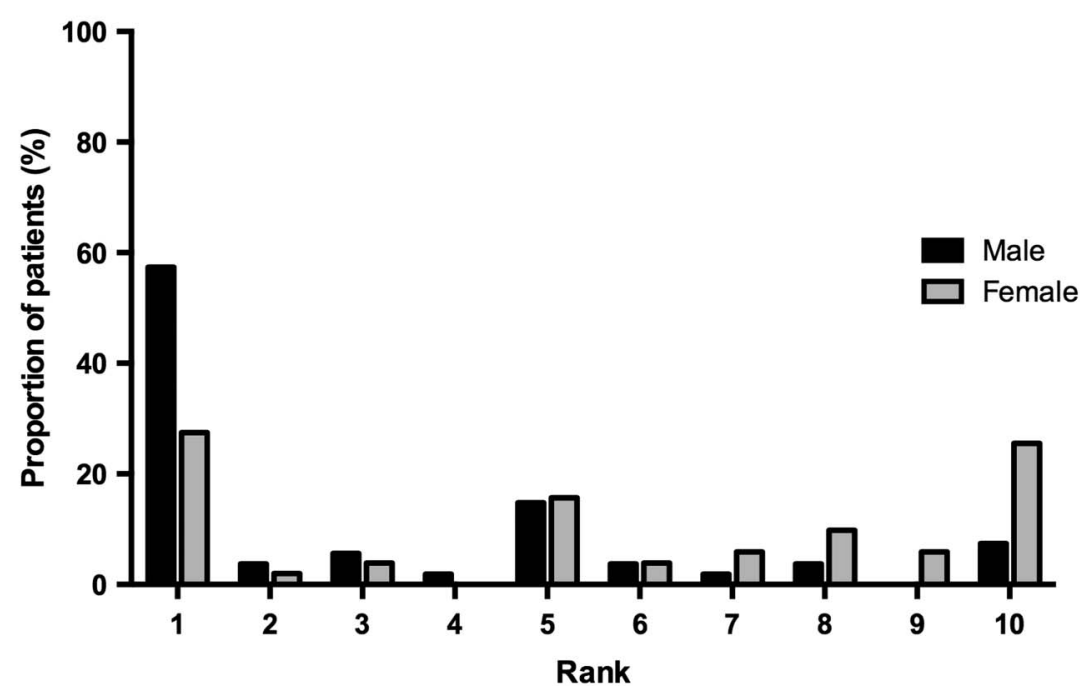

Figure 3 (A) Visual depiction of male and female rankings of the importance of access to prompt investigations/treatment and the provision of separate male and female accommodation in endoscopy. Symbols on the visual analogue scale represent the median rank for men and women for each feature; whiskers indicate the IQR. Median and modal rankings returned by men and women are provided for each feature. Statistical significance of the difference between median rank reported by men and women for each feature is shown and calculated using the Mann-Whitney $U$ test. (B) Bar chart showing the proportion of male and female patients choosing each rank (1=least important to $10=$ most important) for the importance of provision of single-sex accommodation.

Although most of our patients would be comfortable sharing admission/recovery areas with patients of the opposite sex, a significant proportion (20\%-25\%) reported that they would find this uncomfortable. SSA has previously been reported to be more important to female patients. ${ }^{4} 9$ In keeping with this, approximately $80 \%$ of those reporting disquiet at the prospect of MSA in our endoscopy unit were female. Furthermore, women ranked the importance of SSA provision significantly higher than men. Nevertheless, for women in particular, rankings of SSA importance were strikingly bimodal, with similar numbers of female patients ranking its importance as 1 (least important) and 10 (most important). This reflects the heterogeneity of individual preference encountered in patient populations and highlights the difficulty in prioritising healthcare policy with a 'one size fits all' approach. This may represent differences in views of different age groups since older people are generally less tolerant of MSA; ${ }^{4}$ unfortunately data on age of respondents was insufficient to explore this further.

Although SSA is important to some patients it is often not their primary concern, with other factors allocated greater significance. ${ }^{4}$ Furthermore, many patients have stated that they would be happy to receive inpatient treatment in an MSA area if this facilitated more rapid medical treatment or earlier discharge. ${ }^{9}{ }^{10}$ Similarly, to our patients receiving prompt 
endoscopic investigation and treatment was significantly more important than SSA provision. However, since these are not necessarily mutually exclusive and SSA remains important to a sizeable minority of endoscopy patients, we contend that it should remain the gold standard of care.

Interestingly, most of our patients were unaware of the national and local MSA policies that have been implemented on their behalf. While our results comparing similar cohorts surveyed in 2012 and 2015 demonstrated some success in informing patients about local policies, approximately half remained unaware even in the more recent cohort and there was no change in awareness of broader government policy. This suggests a need for better communication of patients' rights and policies, particularly for those directly affected both at local and national levels.

Several patients were admitted to an alternative area in order to maintain SSA and a significant proportion of these felt their care was compromised as a result. Many UK endoscopy units were designed before introduction of the MSA policy and thus do not have the existing infrastructure to provide separate female and male accommodation concomitantly. Although the ultimate solution is the redesign/rebuilding of units, this is a costly and lengthy process, and we suspect that other units may make similar arrangements (ie, admitting/recovering patients in separate areas to accommodate urgent or specialist procedures) to maintain timely service delivery. Furthermore, those units with multiple single rooms allowing the provision of separate accommodation of men and women pose additional logistical challenges for the nursing staff to safely care for and observe patients, especially after endoscopy. In these situations care must be taken to ensure that the provision of SSA does not impact patient safety. Our analysis of patient experiences in 2012 resulted in improvements in list organisation and consequent improvements in service delivery when audited again 3 years later, indicating that this obstacle is at least partially surmountable through simple improvements in list planning. However, there will inevitably remain urgent cases for whom clinical priority will supersede SSA compliance, and thus 100\% compliance with SSA, though list planning alone is probably unrealistic. At RAEI these cases are managed by admission to an area physically separate from the endoscopy unit; while this maintains formal compliance with MSA policy, for some patients this may have conversely introduced other, negative perceptions or experiences unrelated to privacy/dignity issues.

A limitation of this study is that it reflects the views of a relatively small number of patients in a single endoscopy unit. Patient perceptions might differ in units elsewhere with different socioeconomic, ethnic or cultural demographics. Furthermore, patient experience questionnaires can be difficult to design to ensure unbiased answers. Although we took care to word questions in a neutral way we recognise that biases may nonetheless inadvertently have been introduced. Moreover, as endoscopy staff were responsible for distributing and collecting survey responses patients may have felt obliged to give certain answers. Nevertheless, we took steps to negate these as far as possible and ultimately our data provide interesting insights into patients' views on the importance of MSA in a typical busy district general hospital endoscopy unit.

In conclusion, while segregation of sexes is important to some endoscopy patients, to most prompt provision of services is considerably more important. We must ensure that in providing SSA we do not compromise other aspects of the patient journey and inadvertently diminish patient experience of the service. This may be particularly important, given that most patients remain unaware of the government policy on eliminating MSA. In endoscopy units the competing issues of maintaining privacy and dignity versus delivery of a safe and efficient service must be carefully managed. Otherwise we risk achieving no net gain in patient satisfaction and experience.

Acknowledgements The authors thank the staff at the Royal Albert Edward Infirmary for provision and collection of patient questionnaires and for their stimulating discussions.

Competing interests None declared.

Provenance and peer review Not commissioned; externally peer reviewed.

\section{REFERENCES}

1 Department of Health. Equity and excellence: liberating the NHS. The Stationery Office London, 2010.

2 Commissioning for quality and innovation (CQUIN): February 2014. 2014.

3 Enhancing privacy and dignity: achieving single sex accommodation. NHS Estates 2002.

4 Chief Nursing Officer, Department of Health. Privacy and dignity-a report by the Chief Nursing Officer into mixed sex accommodation in hospitals. 2007.

5 Department of Health. The Operating Framework for the NHS in England 2010/2011. 2010.

6 Beasley C, Flory D. Department of Health, from the Chief Nursing Officer and Deputy NHS Chief Executive. 2010.

7 Department of Health. The Operating Framework for the NHS in England 2012/2013. 2011.

8 Lees L, Buggy G. Promoting single sex acute units. Nurs Times 2010;107:18-20.

9 Bonner HK, Frost SJ, Smith MG, et al. Do patients really mind mixed sex bays in an emergency assessment unit? Acute Med 2009;8:119-22.

10 Bryant D, Adams J. Experience of mixed-sex bays in a general hospital. Nurs Stand 2009;24:41-6.

11 Baillie L. Mixed-sex wards and patient dignity: nurses' and patients' perspectives. Br J Nurs 2008;17:1220-5.

12 Maxwell S, Sigsworth J. Eliminating mixed sex accommodation in hospital to improve patient experience. Nurs Times 2008. 